Monatsschr Kinderheilkd 2010 • 158:834-835

DOI 10.1007/s00112-010-2187-3

Online publiziert: 4. September 2010

(c) Springer-Verlag 2010

\author{
R. Frank ${ }^{1} \cdot$ R. Kerbl ${ }^{2}$ \\ ${ }^{1}$ Gauting \\ ${ }^{2}$ LKH Leoben-Eisenerz, Leoben, Österreich
}

\title{
Depressionen bei Kindern und Jugendlichen
}

tere fachliche Unterstützung und Kooperation mit Spezialisten notwendig.

Inderkinder-undjugendpsychiatrischen Praxis erfolgt eine umfassende Diagnostik, die in ein Behandlungskonzept und eine Koordination der Therapie mündet. Das Risiko von Suizidalität muss immer wieder aufs Neue geprüft werden. Depressive Jugendliche benötigen eine Behandlung über einen längeren Zeitraum. Dem Bericht aus der Kinder- und jugendpsychiatrischen Praxis liegt ein umfassendes Projekt zur Qualitätssicherung zugrunde, insbesondere $\mathrm{zu}$ Ergebnissen der Behandlung und zur Zufriedenheit der Patienten.

Die notfallmäßige Aufnahme in stationäre Behandlung bei einer Krisensituation wie Suizidalität kann zur Klärung einer schon lange bestehenden belastenden Lebenssituation führen. Kinder und Jugendliche in Kliniken sind in ihren Lebensbezügen schwer beeinträchtigt. In Rahmen der multidisziplinären und multimodalen Behandlung kann häufig eine rasche Rückbildung der Symptomatik erzielt werden. Die Weiterführung der Therapie muss in Kooperation mit niedergelassenen Ärzten, Psychotherapeuten, Schule und Jugendhilfe abgestimmt werden.

In der Kinder- und Jugendpsychiatrie wird verbindlich eine standardisierte $B a$ sisdokumentation geführt. Sie beschreibt die wesentlichen diagnostischen und therapeutischen Elemente. Auf der Grundlage routinemäßig erhobener Daten ist ein Vergleich zwischen ambulantem, teilstationärem und stationärem Setting sowie zwischen Institutionen möglich.
Die Auswirkungen der depressiven Erkrankungen von Erwachsenen auf ihre Kinder werden in der Erwachsenenpsychiatrie nicht sehr oft berücksichtigt. Aus einer Kooperation zwischen Psychiatrie und Kinder- und Jugendpsychiatrie entwickelte sich eine in einer psychiatrischen Klinik angesiedelte Sprechstunde für Kinder - SprechKid, die diesen Gehör und Unterstützung anbietet. Für Kinderärzte kann der Aufbau einer Kooperation mit Erwachsenenpsychiatern sehr anregend und unterstützend sein.

\section{(7) Basis aller (psycho-) therapeutischen Ansätze ist eine tragfähige Beziehung}

Für Depressionen stehen wirkungsvolle Behandlungsoptionen zur Verfügung. Grundlage aller (psycho-)therapeutischen Ansätze ist die Entwicklung einer tragfähigen Arzt-Patienten-Beziehung [1], deren Bedeutung in allen Beiträgen herausgestellt wird. Die Wirksamkeit von Psychotherapien ist belegt. Für das Kindesalter liegen nur begrenzt Wirksamkeitsnachweise medikamentöser Behandlungen vor, sodass diese „off label“ durchgeführt werden müssen. Für die Gruppe der selektiven Serotoninwiederaufnahmehemmer (SSRI: „selective serotonin reuptake inhibitor") wurde diskutiert, ob sie eine Erhöhung der Suizidgefahr bewirken. Eine umfassende, methodenkritische Übersichtsarbeit [1] berücksichtigte auch Daten für Kinder und Jugendliche. In ihr wurde der Schluss gezogen, dass bei allen 
antidepressiv wirksamen Medikamenten die erwünschte Verbesserung der depressiven Symptomatik gleichzeitig mit einer Erhöhung des Suizidrisikos einhergehen kann. Nutzen und Gefährdungen der medikamentösen Behandlung sind deshalb im Einzelfall abzuwägen [1]. Nach erfolgreicher Behandlung sollte auf die Möglichkeit von Rückfällen unter Belastungssituationen hingewiesen werden.

Die Beiträge dieses Themenhefts zeigen den Wert standardisierter Daten. Erst im Rahmen der Versorgung lassen sich Nutzen und Anwendbarkeit der unter Forschungsbedingungen festgestellten Wirksamkeit von Behandlungen überprüfen.

Im Kindes- und Jugendalter werden Diagnosen einer Depression nicht sehr oft gestellt. Depressive Verstimmungen sind hingegen häufig anzutreffen. Für Ärzte in Klinik und Praxis ist es eine Herausforderung, Emotionen zu erkennen, sie anzusprechen und damit umgehen zu können. Ein wertschätzender Umgang schafft Vertrauen und stärkt Kinder und Eltern.

Die Beiträge des vorliegenden Themenhefts gehen auf Vorträge auf Münchner kinder- und jugendpsychiatrischen Symposien zurück. Den Autoren gebührt Dank, dass sie ihre Beiträge aktualisiert und ausgearbeitet haben. Der Gastherausgeber dankt Herausgebern und Verlag für die Möglichkeit zur Publikation und für die gute Kooperation. Er verbindet dies mit dem Wunsch, dass die Beiträge in der Praxis von Nutzen sein mögen.

\section{Kan r}

Prof. Dr. R. Frank

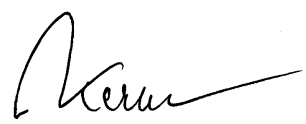

Prof. Dr. R. Kerbl

\section{Literatur}

1. Grawe K, Donati R, Bernauer F (2001) Psychotherapie im Wandel: Von der Konfession zur Profession, 5. Aufl. Hogrefe, Göttingen

2. Möller HJ, Baldwin DS, Goodwin G et al. and the WPA Section on Pharmacopsychiatry (2008) Do SSRIs or antidepressants in general increase suicidality? Eur Arch Psychiatry Clin Neurosci [Suppl 3] 258:3-23

3. Rao U, Hammen CL, Poland RE (2010) Longitudinal course of adolescent depression: neuroendocrine and psychosocial predictors. J Am Acad Child Adolesc Psychiatry 49:141-151

4. Spitz RA (1946) Anaclitic depression: an inquiry into the genesis of psychiatric conditions in early childhood. Psychoanal Study Child 2:113-117

\section{S3-Leitlinie Depression}

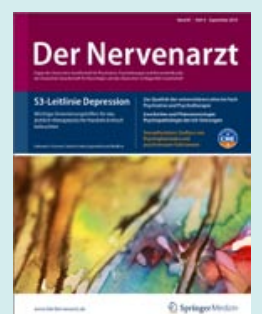

Klinische Leitlinien mit Konzentration auf Diagnose, Behandlung u.a. sind eine wichtige Orientierungshilfe für ärztlich-therapeutisches Handeln. In Zeiten der evidenzbasierten Medizin ist die Entwicklung von Leitlinien elementar für die Psychiatrie, um die Behandlungsqualität der Patienten mit psychischen Erkrankungen nachhaltig zu verbessern. Die Ausgabe 09/10 der Zeitschrift Der Nervenarzt befasst sich mit dem Themenschwerpunkt „S3-Leitlinie Depression“. Das Heft widmet sich der S3-Leitlinie „Unipolare Depression", die auch als Nationale Versorgungsleitlinie entwickelt wurde. Wichtige Punkte werden aus verschiedenen Perspektiven dargestellt, ihre Anwendung wird kritisch beleuchtet und ein Ausblick in die Zukunft der Leitlinienentwicklung wird gegeben. Unter anderem werden folgende Themen behandelt:

- Leitlinienintentionen der DGPPN

- Evidenzbasierte Therapie der Depression

- Wenn Leitlinie auf Versorgungswirklichkeit trifft

- Das Konzept der Entwicklung von S3-Leitlinien

Bestellen Sie diese Ausgabe zum Preis von EUR 33,- zzgl. Versandkosten bei: Springer Customer Service Center GmbH Kundenservice Zeitschriften

Haberstr. 7

69126 Heidelberg

Tel.: +49 6221-345-4303

Fax: +49 6221-345-4229

leserservice@springer.com www.DerNervenarzt.de

\section{Korrespondenzadresse \\ Prof. Dr. R. Frank \\ Flurstraße 24, 82131 Gauting \\ Reiner_Frank@web.de}

